\title{
Dasatinib-Induced Colitis with Rectal Sparing in a Patient with Chronic Myeloid Leukemia (Chronic Phase) on Dasatinib as an Upfront Therapy: Case Report
}

\author{
Zakaria Maat $^{\mathrm{a}}$ Kamran Mushtaq $^{\mathrm{b}}$ Mohamed A. Yassin $^{\mathrm{c}}$ \\ a Department of Medical Education, Hamad Medical Corporation, Doha, Qatar; ${ }^{b}$ Department \\ of Gastroenterology, Hamad Medical Corporation, Doha, Qatar; 'Department of \\ Hematology and BMT, Hamad Medical Corporation, Doha, Qatar
}

\section{Keywords}

Chronic myeloid leukemia · Dasatinib · Colitis

\section{Abstract}

Dasatinib is a BCR-ABL tyrosine kinase inhibitor which was approved in 2006 for the treatment of adults diagnosed with Philadelphia chromosome-positive $(\mathrm{Ph}+)$ chronic myeloid leukemia $(C M L)$ in chronic phase $(C P)$ and accelerated (myeloid or lymphoid blast) phase and CML with resistance or intolerance to prior therapy including imatinib and in adults with $\mathrm{Ph}+$ acute lymphoblastic leukemia. Common adverse reactions $(>15 \%)$ in patients diagnosed with $\mathrm{CP}-\mathrm{CML}$ include myelosuppression, fluid retention, and diarrhea. We report a 34-year-old Filipino female patient who received dasatinib as upfront therapy for the treatment of CP-CML who experienced chronic diarrhea for 2 months, which progressed to colitis.

\section{Introduction}

Newly diagnosed chronic-phase chronic myeloid leukemia (CP-CML) can be treated with imatinib where about two-thirds of patients could benefit from imatinib as upfront therapy [1]. Many trials of new-generation medications are in progress, with the aim of improving 
standard therapy. A range of assessments are available for evaluating novel strategies [2]. Based on existing evidence, achieving a complete cytogenetic response (CCyR) within 12 months provides the best prediction for longer term benefit, although durable response and freedom from disease progression are the main treatment goals. First-line treatment with dasatinib $100 \mathrm{mg}$ once daily or nilotinib $300 \mathrm{mg}$ twice daily resulted in high rates of CCyR and major molecular response (MMR) compared with previous data [3]. The production and analysis of new data from next-generation agents and modified imatinib-based strategies are likely to be an important debatable area in the next few years. Therefore, it is very important to report the adverse effects associated with the use of these medications since it may affect treatment decision by the patient or the prescribing physician [4]. Previous studies have indicated that immunological mechanisms can affect colonic mucosa in patients receiving dasatinib which can cause hemorrhagic colitis.

\section{Case Report/Case Presentation}

A 34-year-old Filipino female not known to have any chronic illness and no positive family history of malignancy presented to ED complaining of left lower limb pain of days duration. Her CBC showed significant leukocytosis with mild anemia and thrombocytosis. Peripheral smear was suggestive of CML, and she was then referred to the hematology clinic at the National Center for Cancer Care and Research. Bone marrow aspiration as well as cytogenetic studies and BCR/ABL by PCR confirmed the diagnosis of CML in CP.

Her clinical examination was not remarkable, with no hepatomegaly, the spleen was palpable 3 fingers below costal margins, and no palpable masses. Treatment options as well as both pros and cons for each therapy were explained and discussed with the patient. The patient opted for dasatinib as upfront therapy, which was started as $100 \mathrm{mg}$ orally once daily, and the patient was followed up as per European Leukemia Net (ELN) guidelines. The dose was reduced to $70 \mathrm{mg}$ orally 7 months later because of hematological toxicity. She achieved CHR, CCyR, and MMR after receiving dasatinib for 27 months. However, recently, she started to have bloody diarrhea for the past 2 months. The patient was admitted to the hospital for further evaluation and management. Her workup showed normal hemogram and normal serum concentrations of creatinine, urea, hepatic enzymes, and electrolytes on admission. Colonoscopy showed patchy erythema and inflammation in the sigmoid colon with rectal sparing, and retroflexion revealed small anal papillae (shown in Fig. 1, 2).

Biopsy showed large bowel mucosal fragments with no significant pathological changes. Stool microbiology as well as the clostridium toxin assay was negative.

\section{Discussion}

CML is a myeloproliferative neoplasm that is associated with the Philadelphia chromosome $\mathrm{t}(9 ; 22)(\mathrm{q} 34 ; \mathrm{q} 11)$ and the BCR-ABL fusion gene, which produces a constitutively active $\mathrm{BCR}-\mathrm{ABL}$ tyrosine kinase. $\mathrm{CP}-\mathrm{CML}$ can progress from a relatively indolent disorder to accelerated phase (AP) or blast crisis (BC).

The goals for management of CP-CML are to achieve clinical remission, maintain longterm disease control, and avoid disease progression to AP or BC, while optimizing quality of life by limiting treatment-related toxicity. For patients who achieve a sustained and deep molecular remission, a trial of discontinuation of therapy to achieve a treatment-free remission is a long-term goal of management.

\section{Karger'}


Fig. 1. Sigmoid colon showing patchy erythema and inflammation.

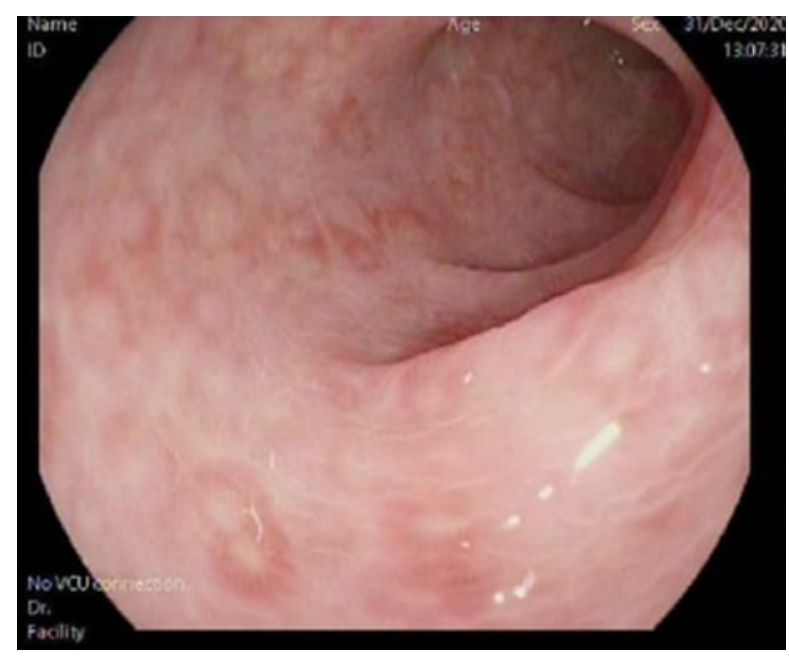

Fig. 2. Sigmoid colon showing patchy erythema and inflammation.

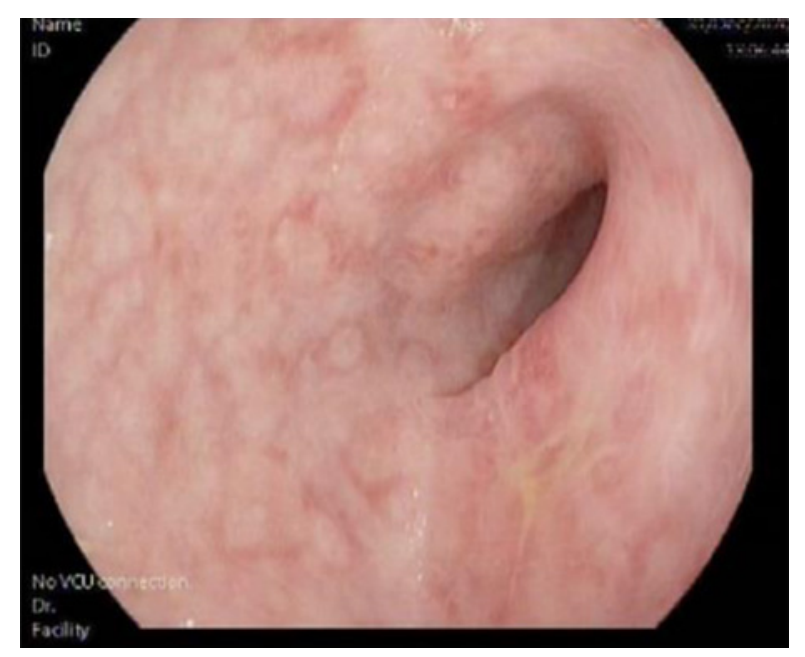

Pretreatment evaluation should include history, physical examination, specific laboratory studies, and determination of CML risk category (using either the Sokal, Euro, EUTOS, or ELTS tools). With the exception of CML diagnosed during pregnancy, BCR-ABL tyrosine kinase inhibitor (TKI) is recommended for initial treatment of all patients with CP-CML, based on superior treatment outcomes and modest toxicity compared with other treatments (e.g., cytotoxic agents, interferons, and hematopoietic cell transplantation). BCR-ABL TKI is selected according to the CML risk score and the goal of achieving a treatment-free remission. No individual TKI is preferred for initial treatment of all patients with CP-CML. The choice of a particular TKI must also consider the side effect profile, comorbid illnesses, availability, cost, and patient preference. For patients with high-risk CP-CML, the recommended treatment is with a second-generation (2G) TKI (e.g., nilotinib, dasatinib, and bosutinib), when available, rather than imatinib. Progression to AP/BC is a concern in patients with high-risk CML, and compared with imatinib, 2 G TKIs may be associated with lower rates of disease progression. However, imatinib and 2G TKIs produce comparable rates of overall survival, progression-free survival, and adverse events in high-risk CML.

Dasatinib is a second-generation TKI that is associated with cytopenias, pleural effusions, other fluid retention, QT prolongation, and bleeding. It should not be given to patients with hypokalemia, hypomagnesemia, or who have or may develop prolongation of the QTc interval, 
and generally avoidance of dasatinib is advised in patients with a bleeding history, pleural effusion, and heart failure. Patients should be screened by EKG for QTc interval at baseline, and hypokalemia or hypomagnesemia should be corrected before starting the treatment.

Dasatinib treatment of CML has been also associated with colitis in a few cases [5]. One case was reported by Patodi et al. [6] in a 59-year-old male with CP-CML, who received dasatinib as a second-line treatment, and the event happened 3 years after the initiation of dasatinib. Another case was reported by Karima et al. [7] in a 36-year-old male with CML transforming to $\mathrm{BC}$ acute myeloid leukemia who received dasatinib at a dose of $140 \mathrm{mg}$ orally once daily after doxorubicin adriamycin or 3-plus-7 protocol on day 34 of treatment. Our patient has received dasatinib as upfront treatment of CML CP and colitis happened, so it seems that dasatinib triggered the colitis. However, few days after stopping dasatinib treatment, significant improvement occurred.

The phase of CML appears to have a relation with colitis. Colitis has been described during the $\mathrm{BC}$ of CML after imatinib failure and in CP-CML when it was used as upfront treatment. However, colitis was not reported during the AP or blast lymphoid phase of the disease. One report looking at bleeding diathesis in CML patients receiving dasatinib therapy noted that $81 \%$ of all bleeding episodes were confined to the gastrointestinal tract [8]. Dasatinib, which is eliminated in the feces, is consistent with gastrointestinal bleeding. Therefore, after being exposed to dasatinib, the lower gastrointestinal tract may be the most vulnerable during dasatinib elimination, and this may explain the occurrence of colitis in our patient.

After stopping dasatinib treatment, our patient was started on imatinib; pros and cons were explained, and her questions were addressed as well; we encouraged the patient for treatment adherence in case of any adherence difficulty or problem (e.g., economic factor and medication side effect) and follow-up for close monitoring and dose adjustment since it can affect the treatment success $[9,10]$. Our group is studying the unmet clinical needs in Myeloproliferative neoplasms [11] and CML like cost effective analysis for second generations TKIs when used as upfront [12], the association of tuberclosis with CML [13], the reactivation of hepatitis B with CML [14], ophthalmic manifestations as initial presentation in patients with CML [15], Effects of intermittent fasting on CML [16], autoimmune hemolytic anemia and its association with different therapies in CML [17], priapism [18, 19] and male fertility [20], as well as obesity [21] and obesity related surgeries in patients with CML [22].

\section{Conclusion}

Patients with CML receiving dasatinib for chronic or blast phase may develop prolonged bloody diarrhea which should be taken seriously.

\section{Statement of Ethics}

This article was approved by the Hamad Medical Corporation Medical Research Center. This research was conducted ethically in accordance with the World Medical Association Declaration of Helsinki. The subject in this study has given informed consent in written form to publish this case.

\section{Conflict of Interest Statement}

The authors have nothing to disclose.

\section{Karger'}




\section{Case Reports in Oncology}

\begin{tabular}{l|l}
\hline Case Rep Oncol 2021;14:1441-1446 \\
\hline DOI: 10.1159/000516794 & $\begin{array}{l}\text { @ 2021 The Author(s). Published by S. Karger AG, Basel } \\
\text { www.karger.com/cro }\end{array}$ \\
\hline
\end{tabular}

Maat et al.: Dasatinib-Induced Colitis with Rectal Sparing in a Patient with CML

\section{Funding Sources}

This article was funded by the Qatar National Library.

\section{Author Contributions}

Case identification and eligibility assessment were done by Zakaria Maat. Critical review of the manuscript and revisions was done by Mohamed A. Yassin. Initial manuscript writing was by Zakaria Maat. Clinical review of the manuscript in gastroenterology part was done by Kamran Mushtaq. All authors performed literature review and reviewed and gave approval of the final manuscript.

\section{Data Availability Statement}

Data available upon request from authors.

\section{References}

1 Steinberg M. Dasatinib: a tyrosine kinase inhibitor for the treatment of chronic myelogenous leukemia and Philadelphia chromosome-positive acute lymphoblastic leukemia. Clin Ther. 2007;29:2289-308.

2 Yassin MA, Nashwan AJ, Soliman AT, Yousif A, Moustafa A, AlBattah A, et al. Cytomegalovirus-induced hemorrhagic colitis in a patient with chronic myeloid leukemia (chronic phase) on dasatinib as an upfront therapy. Clin Med Insights Case Rep. 2015 Jan; 8:77.

3 Talpaz M, Shah NP, Kantarjian H, Donato N, Nicoll J, Paquette R, et al. Dasatinib in imatinib-resistant Philadelphia chromosome-positive leukemias. N Engl J Med. 2006;354:2531-41.

4 Turkina A, Wang J, Mathews V, Saydam G, Jung CW, Al Hashmi HH, et al. TARGET: a survey of real-world management of chronic myeloid leukaemia across 33 countries. Br J Haematol. 2020 Sep;190(6):869-76.

5 Riaz LM, Galal KM, Hussein RM, Nashwan AJ, Yassin MA. Dasatinib-induced colitis in a patient with chronic myeloid leukemia (chronic phase). Int J Case Rep. 2018;3:43.

6 Patodi N, Sagar N, Rudzki Z, Langman G, Sharma N. Haemorrhagic colitis caused by dasatinib. Case Rep Hematol. 2012;2012:417106.

7 Kmira Z, Nesrine BS, Houneida Z, Wafa BF, Aida S, Yosra BY, et al. Severe hemorrhagic colitis in a patient with chronic myeloid leukemia in the blastic phase after dasatinib use. World J Gastrointest Pathophysiol. 2013 Aug 15;4(3):59-62.

8 Christopher J, Cui D, Wu C, Luo R, Manning JA, Bonacorsi SJ, et al. Metabolism and disposition of dasatinib after oral administration to humans. Drug Metab Dispos. 2008;36:1357-64.

9 Al-Dewik NI, Morsi HM, Samara MM, Ghasoub RS, Gnanam CC, Bhaskaran SK, et al. Is adherence to imatinib mesylate treatment among patients with chronic myeloid leukemia associated with better clinical outcomes in Qatar? Clin Med Insights Oncol. 2016 Jan;10:95.

10 Al-Dewik NI, Jewell AP, Yassin MA, El-Ayoubi HR, Morsi HM. Molecular monitoring of patients with chronic myeloid leukemia (CML) in the state of Qatar: optimization of techniques and response to imatinib. QScience Connect. 2014 Mar 1;2014(1):24.

11 Yassin MA, Taher A, Mathews V, Hou HA, Shamsi T, Tuğlular TF, et al. MERGE: a multinational, multicenter observational registry for myeloproliferative neoplasms in Asia, including Middle East, Turkey, and Algeria. Cancer Med. 2020 Jul;9(13):4512-26.

12 Adel A, Abushanab D, Hamad A, Abdulla M, Izham M, Yassin M. Assessment of dasatinib versus nilotinib as upfront therapy for chronic phase of chronic myeloid Leukemia in Qatar: a cost-effectiveness analysis. Cancer Control. 2021;28:10732748211001796.

13 Iqbal P, Soliman A, De Sanctis V, Yassin MA. Association of tuberculosis in patients with chronic myeloid leukemia: a treatment proposal based on literature review. Expert Rev Hematol. 2021;14:211-7.

14 Atteya A, Ahmad A, Daghstani D, Mushtaq K, Yassin MA. Evaluation of Hepatitis B reactivation among patients with chronic myeloid leukemia treated with tyrosine kinase inhibitors. Cancer Control. 2020;27(1): 1073274820976594.

15 Yassin MA, Ata F, Mohamed SF, Alkhateeb A, Naeem U, Al-Qatami AI, et al. Ophthalmologic manifestations as the initial presentation of chronic myeloid leukemia: a Review. Surv Ophthalmol. 2021;S0039-6257-2. 
16 Yassin MA, Ghasoub RS, Aldapt MP, Abdulla MA, Chandra P, Shwaylia HM, et al. Effects of intermittent fasting on response to tyrosine kinase inhibitors (TKIs) in patients with chronic myeloid leukemia: an outcome of European leukemia net project. Cancer Control. 2021;28:10732748211009256.

17 Hamamyh T, Yassin MA. Autoimmune hemolytic anemia in chronic myeloid leukemia. Pharmacology. 2020; 105:630-8.

18 Ali E, Soliman A, De Sanctis V, Nussbaumer D, Yassin M. Priapism in patients with chronic myeloid leukemia (CML): a systematic review. Acta Biomed. 2021;92(3):e2021193.

19 Ali EA, Nashwan AJ, Yassin MA. Essential thrombocythemia with (type 2) calreticulin presented as stuttering priapism case report and review of literature. Clin Case Rep. 2021;9:399-404.

20 Yassin MA, Soliman AT, De Sanctis V. Effects of tyrosine kinase inhibitors on spermatogenesis and pituitary gonadal axis in males with chronic myeloid leukemia. J Cancer Res Ther. 2014;2:116-21.

21 Abdulla AJ, Chandra P, El Akiki S, Sorio C, Tomasello L, Boni C, et al. Clinicopathological variables and outcome in chronic myeloid leukemia associated with bcr-abl1 transcript type and body weight: an outcome of European leukemia net project. Cancer Control. 2021;28.

22 Yassin MA, Kassem N, Ghassoub R. How I treat obesity and obesity related surgery in patients with chronic myeloid leukemia: an outcome of an ELN project. Clin Case Rep. 2021;9:1228-34. 